\title{
La obra de Amartya Sen
}

\author{
Josep Maria VEGARA CARRIó \\ Universidad Autónoma de Barcelona y Universidad Pompeu Fabra \\ Josepmaria.vegara@uab.cat
}

Received: 21/03/2016

Accepted: 09/09/2016

\section{Resumen}

El objetivo del presente trabajo es presentar los grandes rasgos de la obra global de Amartya Sen. Sen nació el año 1933 en Santiniketan, en Bengala occidental (India). Realizó estudios universitarios en Calcuta y posteriormente en Cambridge (Reino Unido). Ha ejercido sucesivos puestos docentes universitarios en la India, Inglaterra y los Estado Unidos. Sen ha presidido diversas Asociaciones de economistas; asimismo ha influido en instituciones internacionales como el Banco Mundial o en diversas agencias de las Naciones Unidas. Recibió el Premio Nobel de Economía en 1998 por su contribución a la economía del bienestar.

No es de extrañar que la extensa obra de Sen ocupe un merecido y relevante lugar en el campo de la economía política y que haya generado asimismo una notable polémica en el campo de la filosofía política. Sus contribuciones económicas cubren un amplio temario, de la teoría de la elección social a las desigualdades, desarrollando metodologías para su medición. Ha cubierto también los campos de la economía del bienestar y de la teoría de la justicia, polemizando especialmente con John Rawls. Su obra, en conjunto, gravita en torno a los problemas del desarrollo económico, desde una perspectiva global y explícita; así, en su libro de síntesis sobre el tema -Development as Freedom, publicado en 1999, indica que en su enfoque "la expansión de la libertad es tanto el fin primordial del desarrollo como su medio principal".

Su formación filosófica le permite tratar con rigor temas inhabituales en los escritos de un economista o bien le facilita incorporar dimensiones o reflexiones filosóficas a su discurso económico; le permite hacerlo y también le induce a hacerlo, sin duda. Este hecho puede sorprender por cuanto lo más habitual es que la componente filosóficaética -o ideológica, si se prefiere- se halle implícita, oculta en un relato de contenido aparentemente tan sólo analítico y neutral.

Palabras clave: historia del pensamiento económico, equidad, justicia, desigualdad, análisis de la toma de decisiones colectiva, desarrollo económico, innovación, cambio técnico, crecimiento

Clasificación JEL: B2, D63, D7

\section{The Works of Amartya Sen}

\section{Abstract}

The goal of this paper is to present the works of Amartya Sen. Sen was born the year 1933 in Santiniketan, West Bengala (India). He studied at Calcutta University and later in Cambridge (United Kingdom). He has been teaching in different Universities in India, United Kingdom and the United States. He has also been President of different professional associations and has influenced in several international institutions such as the World Bank and different UN agencies. He received in 1998 the Nobel Prize in Economics for his contributions in the field of welfare economics.

It's no wonder that his extensive work occupies a fully deserved place in the field of political economy and has also generated a considerable controversy in this of political philosophy. His economic contributions cover a broad agenda, from social choice theory to inequalities, including methodologies for their measurement. He has also covered the fields of welfare economics and the theory of justice, arguing with John Rawls. His work, globally considered, gravitates around the problems of economic development, from a global and explicit perspective; so, in his book on the subject synthesis -Development as Freedom, published in 1999, Sen indicates that "the expansion of freedom in his approach is both the primary purpose of development and the primary means".

His philosophical background allows him to deal rigorously non-current topics for economists or to incorporate philosophical thoughts in an economic discourse. This aspect may be surprising because it is common that the philosophical-ethical dimension -the ideological dimension- is implicit, non-visible in a text apparently analytical and neutral.

Keywords: history of economic thought, equity, justice, inequality, analysis of collective decisions making, economic development, innovation, technological change, growth

JEL Classification: B2, D63, D7 


\section{Introducción}

Amartya Sen es un economista singular, no sólo por su amplio reconocimiento en el seno de la profesión sino también por la amplitud de los temas que ha tratado y la dimensión filosófica de su pensamiento. En este trabajo efectuaremos un recorrido a lo largo de su obra económica, analizando también su dimensión filosófica cuando esta posea implicaciones sobre la elección de temas, como ocurre cuando trata temas de política económica, donde cobra especial relevancia su concepción del papel de la ética en el comportamiento individual así como su idea de justicia; exploraremos pues sus posiciones pero también sus motivaciones, y prestaremos también atención a las polémicas en las que participado activamente.

\section{Biografía}

Amartya Sen nació el año 1933 en Santiniketan, en Bengala occidental (India); estudió en una escuela fundada por el poeta Rabindranath Tagore (VV.AA. 1999a y 1999b). Después de la separación de Pakistán de la India -ocurrida en 1947, en medio de violentos enfrentamientossu familia se desplazó a vivir a la India. Durante su infancia Sen fue testigo de las consecuencias de una gran hambruna en 1943 que causó entre dos y tres millones de muertos, así como también de la violencia entre hindúes y musulmanes, poco antes de la partición de la India y Pakistán; fueron dos experiencias que le marcaron profundamente.

Realizó estudios universitarios en Calcuta y obtuvo un Bachillerato de Artes en Economía. Posteriormente se instaló en Cambridge (Reino Unido) donde obtuvo un segundo Bachillerato de Artes en Economía y también el doctorado, en 1959, con una tesis sobre "La selección de técnicas" bajo la supervisión de Joan Robinson. Durante este período también cursó estudios de filosofía. En aquellos años, el debate económico en Cambridge gravitaba fundamentalmente en torno a las distintas interpretaciones de la obra de Keynes, con protagonistas como Richard Kahn, Nicholas Kaldor y Joan Robinson en un bando y Dennis Robertson, Harry Johnson, Peter Bauer y Michael Farrell en el otro. Entre otros profesores tuvo a la radical Joan Robinson, al marxista Maurice Dobb y también a Piero Sraffa. Durante estos años sus posiciones políticas sin ser militante de ninguna organización política- reflejaban una opción por el socialismo, caracterizado por la propiedad estatal en el sector industrial y por cooperativas en el sector agrario (VV.AA., 2013), como había explicado con detalle en un artículo publicado en 1959, "Why planning?" y se refleja en su obra de 1960, Choice of Techniques; posteriormente evolucionó hacia posiciones socialdemócratas singulares.

Ha tenido sucesivos puestos docentes universitarios a lo largo de su vida académica: primero en la Universidad de Calcuta (1956-1958) como profesor de Economía; posteriormente regresó a Cambridge -donde presentó su tesis- y estuvo en el MIT entre 1960 y 1962, para luego instalarse en la Universidad de Delhi (1963-1971) como profesor de Economía; ha enseñado asimismo en el Nuffield College (1977-1980) y en el All Souls College (1980-1988), ambos de la Universidad de Oxford; en la London School of Economics (1971), en el Trinity College de la Universidad de Cambridge (1957-1963 y 1997-2004) y en la Universidad de Harvard como profesor de Economía y de Filosofía (1987-1998 y desde 2004).

Su carrera profesional ha sido académica y recibió el Premio Nobel de Economía en 1998 por "su contribución a la economía del bienestar". ${ }^{1}$ Muy activo a nivel institucional, ha sido presidente de la Econometric Society (1984), la Indian Economic Association (1989), la American Economic Association (1994) y la International Economic Association (1986-1989).

\section{La obra}

En 1960 publicó el libro Choice of Techniques. An Aspect of the Theory of Planned Economic Development, basado en su tesis doctoral. El tema de la selección de técnicas es calificado por Sen como de uno de los problemas más importantes de la planificación del desarrollo puesto que las distintas tecnologías implican a menudo diferentes estrategias, con efectos muy diferentes sobre las prestaciones de la economía. El marco institucional que el autor considera incorpo-

\footnotetext{
${ }^{1}$ Véase su discurso de recepción del Premio Nobel (Sen, 1999a).
} 
ra: (a) propiedad pública de los medios de producción; (b) la coordinación central de los planes de las diversas industrias, y (c) la ausencia de control del consumo. No obstante, Sen no especifica el papel de los mercados como mecanismo institucional de coordinación; por otra parte, el trabajo no contiene ninguna referencia al debate sobre los problemas del cálculo económico en una economía socialista.

El tema central se refiere a la elección de la intensidad de capital en el marco de una economía formada por dos sectores y dos mercancías. El primer sector es el tradicional, productor de trigo. El segundo sector - a planificar- puede bien producir máquinas utilizando sólo trabajo o bien puede producir trigo utilizando máquinas y trabajo. Existe una oferta ilimitada de trabajo. Sen comenta brevemente que el problema de la medición del capital plantea problemas bien conocidos y -puesto que desea evitarlos- tratará el capital como homogéneo, considerando que existe sólo un tipo de máquinas.

Más tarde, en 1970, publicó una selección de artículos sobre economía del crecimiento que incluía las contribuciones iniciales de Harrod y de Domar, así como las de los neoclásicos y los post-keynesianos. ${ }^{2} \mathrm{Y}$ en 1974 publicó su toma de posición sobre la controversia del capital en "On Some Debates in Capital Theory", Economica, debate con el que se había familiarizado durante sus años de estancia en Cambridge. Utilizando un estilo literario poco frecuente en economía, presenta una fábula cargada de ironía, en la que Buda es uno de los protagonistas y un desolado personaje -Subhuti- pide a Buda que haga todos los bienes de capital homogéneos, para eliminar los problemas suscitados por Sraffa sobre la medición del capital y sus consecuencias críticas. Sen se manifiesta claramente de acuerdo con las críticas sraffianas pero ello no es obstáculo para que su referencia a Solow -principal antisraffiano- sea respetuosa, pues afirma que el Venerable Solow puede hacer supuestos peculiares pero nunca comete errores (p.30).

\section{Cambio de tema: de la teoría del desarrollo a la teoría de la elección social}

Durante los años sesenta Sen inició un cambio temático importante en la dirección de la teoría de le elección social. Según cuenta él mismo, poco después de la publicación del libro de Kenneth Arrow Social Choice and Individual Values, en 1951, un compañero de estudios Sukhamoy Chakravarty- le puso en la pista del libro pero Sen tardó algún tiempo en poder dedicarse al tema, sobre el que publicó el artículo "The Impossibility of a Paretian Liberal" en 1970, el mismo año en el que también publicó el libro Collective Choice and Social Welfare. Según explica él mismo, Dobb le apoyó pero no lo hicieron ni Sraffa ni Robinson.

En "The Impossibility of a Paretian Liberal" Sen presenta una primera toma de posición sobre los procedimientos de elección social; en concreto, analiza una objeción muy común que sostiene que la regla de la mayoría para la toma de decisiones colectivas no es liberal respecto de las decisiones privadas e ilustra dicho carácter con el siguiente ejemplo: "si usted prefiere tener un muro rosa a uno blanco, la sociedad debería permitirlo, incluso si la mayoría de la comunidad prefiriera verlo blanco" (1970, p. 152). Se trata pues de una objeción relativa a los campos de aplicación de la regla de la mayoría en las decisiones colectivas.

En el artículo de 1976 "Rational Fools: A Critique of the Behavioral Foundations of Economic Theory", Sen destaca que la línea de pensamiento utilitarista que arranca con Edgeworth pone el acento en el interés personal-propio (self-interest) como motivación del comportamiento individual y, según su propio criterio, constituye un enfoque innecesariamente limitado; y para corregirlo, introduce los conceptos de simpatía y de compromiso. ${ }^{3}$ La simpatía -en la línea de Adam Smith de la Teoría de los sentimientos morales- corresponde al caso en que:

La preocupación por los demás afecta directamente al propio bienestar. Si el conocimiento de la tortura de otros te enferma, se trata de simpatía; si no te hace sentir personalmente peor pero piensas que es malo y estás dispuesto a hacer algo para pararlo, es un caso de compromiso [...]. El compromiso, por supuesto, se halla estrechamente relacionado con la moral propiamente dicha. Entendiendo moral en

\footnotetext{
${ }^{2}$ Sen A. 1970. Growth economics. Harmondsworth, Middlesex, Penguin Modern Economics Reading.

${ }^{3}$ La expresión original es commitment.
} 
un sentido amplio que engloba una variedad de influencias, de las religiosas a las políticas, de las mal comprendidas a las bien argumentadas. Es más, la característica del compromiso que me concierne más directamente es la que señala que éste constituye una cuña entre la elección personal y el bienestar personal, identidad en la que buena parte de la teoría económica tradicional confía [...]

Así pues, "la conexión básica entre los comportamientos de elección y los logros de bienestar en los modelos de bienestar, se quiebra tan pronto como el compromiso se admite como un ingrediente de la elección" (1976, pp. 89-91) pues éste puede conducir a empeorar el bienestar personal de quien elige.

En definitiva, la elección basada exclusivamente en el bienestar propio -en el sentido estricto- no permite analizar adecuadamente toda la gama de comportamientos. La relevancia de estas precisiones para la economía derivan de que en ésta no existen únicamente los bienes privados conseguibles en los mercados sino que existen asimismo problemas de suministro de bienes públicos que pueden estar sujetos a comportamientos de free riders, de polizones.

En 1971 publicó Collective Choice and Social Welfare. ${ }^{4}$ La obra constituye un manual avanzado sobre el tema, con novedades teóricas y organizado en torno a una sucesión de capítulos, unos con formulaciones matemáticas y otros no formalizados. En el prefacio Sen afirma que la teoría de la elección social pertenece a varias disciplinas y que no puede ser tratado adecuadamente dentro de los límites de la economía, a pesar de que constituya un aspecto crucial propio. Destaca su conexión con la economía del bienestar, la teoría de la planificación y la economía pública, así como su conexión con la ciencia política y la filosofía. Al referirse a los colegas a los agradece su colaboración, en primer lugar destaca a Maurice Dobb y a Kenneth Arrow.

El libro constituye una síntesis del campo, al mismo tiempo que -al relajar los supuestos iniciales formulados por Arrow y que conducían al "teorema de imposibilidad"- obtiene diversas reglas viables de elección social. El libro fue precedido por un curso que dio en la Universidad de Harvard, compartido con Kenneth Arrow y con John Rawls, dos referentes clave en la obra de Sen. Entre otros temas, Sen analiza los sistemas de votación, las relaciones existentes entre la ética y las preferencias, el papel de los juicios de valor, la paradoja liberal, la comparación interpersonal de utilidades y el principio del maximin propuesto por Rawls.

La toma de posición más reciente de Sen sobre la elección social puede verse en el libro colectivo de 2014 de E. Makin y A. Sen, The Arrow Impossibility Theorem (Columbia-New York, Chester West Sussex, Columbia University Press).

En su revisión de las conclusiones alcanzadas por Arrow, a finales de los años sesenta, Sen había avanzado ya ciertas críticas al marco estrictamente utilitarista de la teoría económica, y señalaba la interrelación existente entre la economía y la ética, discrepando del pretendido objetivismo característico de la noción de preferencias revelada; en este periodo es muy notable la conexión existente entre los trabajos de John Rawls, Kenneth Arrow y el propio Amartya Sen. Es destacable que en la primera edición de una teoría de la justicia de John Rawls aparezcan en muy diversos lugares referencias a la obra de Sen.

\section{De la elección social a las desigualdades, el hambre y la pobreza}

Sen ha precisado que su línea de trabajo sobre las desigualdades fue inspirada por la obra de Anthony Atkinson. En 1973 Sen publicó On Economic Inequality que, años después, fue seguido por Inequality Reexamined en 1992, ${ }^{5}$ obra que examinaremos más adelante. Sus contribuciones publicadas durante los años setenta y ochenta están recogidos en dos recopilaciones de artículos: Choice, Welfare and Measurement (1982) y Resources, Values and Development (1984). El primero incluye el artículo "Equality of What?", publicado en $1979^{6}$ y que marca un inicio de línea relativa las desigualdades. A mediados de los años setentas inició su línea de

\footnotetext{
${ }^{4}$ Sen, A. 1970. Collective Choice and Social Welfare. North Holland, Amsterdam, New York, Oxford. 1970

${ }^{5}$ Sen, A. 1992. Inequality Reexamined. New York, Russell Sage Foundation; Oxford, Clarendon Press.

${ }^{6}$ Sen A. 1980. Equality of What? In Sterling McMurrin (ed.) The Tanner Lectures on Human Values, vol. I, University of Utah Press and The Cambridge University Press; reimpreso en 1982. Choice, Welfare and Measurement. Cambridge, Massachusetts, London, Harvard University Press.
} 
trabajo sobre el hambre, en el marco de la Organización Internacional del Trabajo y en colaboración con el economista belga Jacques Drèze con quien publicó Poverty and Famines, entre otras obras. ${ }^{7}$ Por otra parte, Sen trabajó asimismo con Partha Dasgupta y con Stephen Marglin en la metodología de la evaluación de proyectos (Dasgupta, Sen y Marglin, 1972).

\section{6. Ética y economía}

La reflexión sobre la ética constituye una línea permanente en la obra de Sen; una obra singular, monográfica sobre el tema, es On Ethics and Economics publicada en 1987 y que consiguió un notable impacto $;^{8}$ el libro está dedicado a Kenneth Arrow. Sen afirma que no existe justificación alguna para separar el estudio de la economía del de la ética y de la filosofía y que en relación con esta cuestión se plantean dos cuestiones básicas absolutamente fundamentales para la economía. En primer lugar, el tema de la dimensión ética de la motivación humana y, en segundo lugar, el tema de lo que denomina la evaluación del logro social, es decir, la valoración de los resultados agregados de las decisiones y de las políticas.

Sen precisa que su contribución estará centrada en la cuestión de la motivación y el comportamiento económico y, por lo tanto, no en la evaluación de lo que ha denominado el logro social. Indica y reitera que la no consideración explícita de la dimensión ética empobrece el análisis.

Sen destaca que, por sí sola, la consistencia de las diversas opciones o elecciones no puede caracterizar el comportamiento racional. El tema del egoísmo es más complejo que el de la simple consistencia y se plantea por qué debe ser racional únicamente perseguir el propio interés, excluyendo todo lo demás. Según su argumentación, "el egoísmo universal como 'realidad' puede ser falso, pero el egoísmo universal como requisito de la 'racionalidad' es evidentemente absurdo" (1987, p. 3) pues la versión del egoísmo universal excluye cualquier comportamiento racional no egoísta, en contra de lo que afirman numerosos economistas. La evidencia empírica de la existencia de comportamientos no egoístas en el sentido convencional a los que se refiere Sen guarda relación con lo que denomina los grupos intermedios (clases, comunidades, grupos profesionales) y, de forma especial, con los grupos familiares.

Seguidamente Sen plantea, en primer lugar, si las personas se comportan realmente de una manera exclusivamente egoísta y, en segundo lugar, en caso de repuesta afirmativa, si ello genera alguna forma de eficiencia. Sen analiza la posición de Adam Smith sobre estas dos cuestiones y concluye que no existe contradicción entre sus posiciones expuestas en La teoría de los sentimientos morales y en La Riqueza de las Naciones: "Pero el hecho de que Smith observara que el comercio mutuamente beneficioso era muy común no demuestra, en absoluto, que pensara que sólo el egoísmo, o la prudencia, en un sentido amplio, pudieran ser adecuadas para una buena sociedad. No basó la salvación económica en una única motivación” (1987, p. 41).

Sen se refiere a una consecuencia grave generada por el distanciamiento entre la economía y la ética pues ha debilitado el alcance y la importancia de la economía del bienestar. Se refiere al hecho de que al prescindir de las comparaciones interpersonales de utilidad, la economía moderna está vinculada a los límites del "principio de optimalidad" de Pareto y a los dos "teoremas fundamentales del equilibrio general"; Sen indica que el criterio de optimalidad de Pareto es un modo muy limitado de valoración de los logros sociales.

La agencia. Otro aspecto destacado por Sen guarda relación con el tema de lo que denomina la agencia (agency). ${ }^{9}$ El punto de partida consiste en considerar que el éxito de una persona no se puede evaluar considerando de modo exclusivo su bienestar propio, puesto que "una persona puede valorar la promoción de ciertas causas y la existencia de ciertas cosas, aunque la impor-

\footnotetext{
${ }^{7}$ (1981). Otra edición en Sen A., Drèze J. 1989. Hunger and public action. Oxford (England), New York: Clarendon Press Oxford University Press.

${ }^{8}$ Sen A., 1987. On Ethics and Economics. Oxford, Basil Blackwell. Edición española de 1987.

${ }^{9}$ Ocasionalmente, Sen contrapone agente a paciente.
} 
tancia que se atribuya a estos acontecimientos no se refleje en una mejora del bienestar, si es que ésta se produce" (1987, p. 58); ello es así por cuanto en la valoración ética:

Existe una "dualidad" esencial e irreductible en la estimación de una persona. Podemos considerar a la persona en términos de agencia, reconociendo y respetando su capacidad para establecer objetivos, compromisos y valores, etc. y también podemos considerarla en términos de bienestar que, asimismo, demanda atención. Esta dicotomía se pierde en un modelo de motivación exclusivamente egoísta. Sen destaca que incluso es posible que el bienestar de una persona disminuya a causa de la frustración producida por no logar lo que quería conseguir como agente, aun cuando esos logros no estuvieran relacionados directamente con su bienestar. (1987, p. 70)

Subraya asimismo que la introducción de la dimensión de la libertad de la persona y su capacidad de decisión conduce a rechazar no sólo el utilitarismo y el bienestar basado únicamente en su propia utilidad, sino también una serie de enfoques centrados exclusivamente en los logros, es decir, en los resultados obtenidos. ${ }^{10}$

Sen, por otra parte, destaca la importancia de distinguir entre los requisitos de la política institucional, por un lado, y los requisitos de las decisiones personales, por otro. Y asimismo, indica que los temas mencionados tienen un mayor reflejo en las actividades sociales y culturales que en las económicas. Esta línea de pensamiento se refleja en sus reiterados análisis sobre las condiciones de las mujeres en los países en vías de desarrollo.

La tipología de los criterios de decisión. Sen presenta una elaborada clasificación de los diversos criterios de decisión, distinguiendo entre: (a) el bienestar basado en uno mismo; (b) los objetivos basados en el propio bienestar; ( c) la elección basada en los objetivos propios. ${ }^{11}$

(a) El bienestar basado en uno mismo (self centered; oriented welfare), que depende sólo del consumo propio y que no incluye -por lo tanto- ninguna externalidad; así pues, excluye asimismo toda dimensión de simpatía o antipatía y que sí puede darse en los dos tipos siguientes que, en consecuencia, admiten las externalidades;

(b) los objetivos basados en el propio bienestar (self welfare goal) -son pues más amplios que los limitados al simple consumo personal- pero que no valoran de ningún modo el bienestar ajeno;

(c) la elección basada en los objetivos propios (self goal choice), incluidos los objetivos morales, expresados -por ejemplo- mediante normas. Sen discute este caso utilizando el dilema del prisionero. ${ }^{12}$

Esta tipología es clave para captar muchos matices que Sen expresa cuando trata el tema de la toma de decisiones. La selección de artículos de diversos autores publicada en 1982 conjuntamente con Bernard Williams, Utilitarism and beyond, forma parte del mismo proyecto.

\section{Sobre la desigualdad y las capacidades}

Como he indicado ya, el libro publicado en 1992, Inequality Reexamined, es a pesar de su brevedad un referente importante en la obra de Sen sobre las desigualdades. ${ }^{13}$ ¿Por qué la igualdad? ¿E igualdad en términos de qué? Estas son las dos cuestiones básicas, distintas pero interdependientes. Los humanos difieren entre sí de muchas maneras y si pretendemos comparar las ventajas o desventajas respectivas de los individuos, podemos clasificarlas con respecto a muchas variables diferentes para evaluar la desigualdad interpersonal. Una de las variables relevantes para evaluar la igualdad -muy singular, ciertamente- es precisamente la libertad y ello conduce a Sen a precisar, en rigor, que libertad e igualdad no son alternativas excluyentes pues "la liber-

\footnotetext{
${ }^{10}$ Sobre las capacidades y la agencia resulta de interés 2010. "Capability and agency”, capítulo 3 de Morris, Ch. (ed.) (2010).

${ }^{11} 1987$, p. 96. Véase asimismo Sen, 2004. En esta última referencia denomina "self-centered welfare" el bienestar "basado en uno mismo".

${ }^{12}$ Véanse las precisiones que formula Christopher W. Morris (2010, p.29-35) así como la entrevista realizada por Klamer, A. 1989. A Conversation with Amartya Sen, op.cit.

${ }^{13}$ Únicamente 168 páginas de texto y 39 de referencias bibliográficas.
} 
tad se encuentra entre los posibles 'campos de aplicación' de la igualdad y la igualdad se halla entre los posibles 'esquemas' distributivos de la libertad" (1999 [1992], p. 35). Por otra parte, la posición de una persona en la organización social se puede analizar desde dos puntos de vista distintos, el primero relativo a los objetivos alcanzados y el segundo relacionado con la libertad disponible para alcanzarlos (1999 [1992], p. 45). Por libertad Sen se refiere aquí a las oportunidades reales, a las capacidades efectivas de las que disponen las personas para alcanzar sus objetivos.

Sobre el enfoque tradicional -centrado exclusivamente en los logros efectivos o realizaciones- Sen destaca que ha sido cuestionado recientemente por autores como Rawls y Dworkin.

Funcionamientos y capacidad. Sen explica que el bienestar de una persona puede entenderse analizando la calidad de su vida y ésta "puede considerarse como un conjunto de 'funcionamientos' interrelacionados, consistentes en estados y acciones. La realización de una persona puede entenderse como el vector de sus funcionamientos" (1999 [1992], p. 53), que son la clave para la evaluación de su bienestar. Sen, no obstante, no ha proporcionado una enumeración completa de los funcionamientos y de las capacidades que considera relevantes.

La importancia de las capacidades para el bienestar de las personas deriva de dos consideraciones distintas pero interrelacionadas. La primera consiste en que "si los funcionamientos alcanzados constituyen el bienestar de una persona, entonces la capacidad para alcanzar funcionamientos [...] constituirá la libertad de esa persona, sus oportunidades reales para obtener bienestar" (1999 [1992], p. 54); de aquí la relevancia de las capacidades (Sen, 1985). Un ejemplo: disponer de la capacidad de desplazarse tiene unas exigencias diferentes para una persona sin ninguna dificultad para ello que para otra con algún tipo de parálisis; o bien, una persona puede disfrutar de salud pero, si estuviera enferma, ¿a qué servicios de salud tendría acceso? Las capacidades son la clave para evaluar las situaciones, las políticas y la justicia.

La segunda consideración "consiste en hacer depender el propio bienestar alcanzado de la capacidad para funcionar. En sí mismo, el hecho de poder elegir debería entenderse como un componente valioso de la existencia; y una vida de elecciones genuinas con opciones serias puede considerarse, por esta razón, más rica" (1999 [1992], p. 55). Es decir, la propia capacidad de elegir -en el enfoque de Sen- es valiosa en sí misma y no es puramente instrumental. En otras palabras, poder elegir es valioso pues no sólo lo alcanzado es relevante: importa también la libertad de poder alcanzarlo. ${ }^{14}$

Libertad, agencia y bienestar. Las personas consideradas como agentes activos no se guían únicamente por la búsqueda de su propio bienestar: éste un tema recurrente en los escritos de Sen. Por otra parte, al "analizar los objetivos del agente es posible efectuar una distinción ulterior entre, primero, la existencia de aquellas cosas que uno valora y pretende conseguir, y segundo, la existencia de dichas cosas conseguidas por los 'propios' esfuerzos, o en cuya consecución 'uno mismo' ha desempeñado una parte activa”' (1999 [1992], p. 72); es decir, el agente no es pasivo sino que realiza su propio esfuerzo; es pues protagonista.

Las bases de información. Para evaluar la justicia real es preciso disponer de bases de información adecuadas que -Sen precisa- deben referirse, por una parte, a las características personales pertinentes y, por otra, a lo que denomina la forma de combinar las características. Las características pertinentes utilizadas son diversas: las utilidades individuales, las libertades y los bienes primarios (Rawls), los derechos (Nozick), los recursos (Dworkin), las cestas de bienes de consumo (Foley, Varian, Baumol). Las formas de combinarlas son también variadas: el criterio maximin, la suma, la igualdad, etc.

$\mathrm{Al}$ evaluar las desigualdades Sen se pregunta qué conviene juzgar: los logros, o sea, realizaciones, o bien las carencias existentes respecto de los máximos que las personas podrían obtener

\footnotetext{
${ }^{14}$ El ejemplo que plantea Sen es provocador: "Por ejemplo, 'el ayuno' como funcionamiento no es simplemente pasar hambre; es elegir pasar hambre cuando uno tiene otras opciones (1999[1992, p. 65).
} 
(1999 [1992], p. 106). También destaca que la economía del bienestar convencional no ha subrayado el tema de la diversidad humana que resulta clave para su medición y evaluación; la economía del bienestar supone que los ingresos determinan el bienestar social.

Desigualdad y pobreza. La medición de la pobreza tiene dos aspectos: el primero consistente en su identificación y el segundo en proceder a su cómputo global, en proceder a su agregación. Lo primero se realiza tradicionalmente fijando una frontera, una línea de pobreza, de modo que las personas que se sitúan por debajo de la misma se consideran pobres. Sen destaca la importancia de la distribución de los ingresos entre los identificados como pobres y analiza diversos indicadores para ello; ahora bien, como puede anticiparse, para Sen no se trata únicamente de ingresos: en rigor, la pobreza consiste básicamente en una privación, más allá de la simple limitación de los medios disponibles y por ello Sen define su enfoque en términos de fracaso de las capacidades básicas para alcanzar determinados niveles mínimamente aceptables.

En el capítulo siguiente Sen analiza -desde esta perspectiva- las particularidades de diversos grupos especialmente relevantes como es el caso de las mujeres y de determinadas minorías raciales en países desarrollados. Finalmente, examina las defensas alternativas que pueden realizarse de la igualdad en términos de una variable concreta (como la renta o las capacidades); e identifica tres tipos de discusiones ${ }^{15}$ según estén centradas en (1) lo que denomina la identificación del espacio equivocado; (2) el tema de los incentivos o, finalmente (3) la asimetría operativa. Concretamente:

(1) la discusión en términos del espacio equivocado se concentra en señalar que las variables identificadas como claves no son las relevantes para buscar la igualdad. Los otros dos debates están relacionados con la eficiencia.

(2) la discusión en torno a los incentivos es la más frecuente y se refiere a la incidencia de la igualdad sobre las motivaciones individuales relevantes sobre los comportamientos relacionados con el trabajo, el riesgo empresarial y la inversión.

(3) las asimetrías operativas pueden surgir de las diferencias en calificaciones y habilidades que pueden dar lugar a asignaciones diferentes en términos de poder y de capacidades.

El Índice de Desarrollo Humano. La línea de discusión centrada en las bases de información constituye una constante en la obra de Sen. En esta dirección es de destacar que Sen es coautor del diseño del Índice del Desarrollo Humano (IDH) que elaboran desde 1990 las Naciones Unidas y que se halla centrado en tres dimensiones o factores clave de su caracterización, que reciben una determinada ponderación cuantitativa -discutible y discutida, por supuesto- para dar lugar a un índice único por país. El Índice incluye (1) un indicador de salud: la esperanza de vida al nacer; (2) un indicador social: la tasa de alfabetización de los adultos (más la tasa combinada de matriculación en educación primaria, secundaria y superior y los años de duración de la educación obligatoria); y (3) un indicador económico: el Producto Interior Bruto (PIB) medido en paridad del poder adquisitivo en dólares. El IDH ilustra la concepción de Sen sobre las capacidades aplicada al caso de los países en desarrollo. ${ }^{16}$ El PNUD también elabora un Índice específico de pobreza así como diversos indicadores focalizados en torno a las desigualdades de género, una constante en los análisis de Sen.

\section{El discurso de recepción del Premio Nobel en 1998}

La conferencia que pronunció con motivo de la concesión del Premio Nobel, "The Possibility of Social Choice" (1999a), contiene una interesante síntesis de sus posiciones sobre el tema. La cuestión central que motiva la teoría de la elección social la define del modo siguiente:

\footnotetext{
15 "Arguments" en el original inglés se ha traducido en la versión en castellano como "argumentaciones"; es más adecuado utilizar "discusiones".

${ }^{16}$ Véase el resumen del Informe correspondiente al año 2014 en: hdr.undp.org/sites/.../hdr14-summaryes.pdf
} 
¿Cómo puede ser posible conseguir un sistema convincente de juicios agregados sobre la sociedad -por ejemplo, sobre el "bienestar social" o el "interés público" o la "pobreza agregada"- dada la diversidad de preferencias, preocupaciones y dilemas propios de los diferentes individuos "pertenecientes" a la sociedad? ¿Cómo podemos hallar una base racional para para realizar dichos juicios agregados en términos de "la sociedad prefiere esto o aquello" o de "la sociedad debería preferir esto a aquello", o "es esto socialmente correcto"? (1999a, 178)

Sen se remonta a los orígenes de la teoría de la elección social que, como una disciplina sistemática, surgió en tiempos de la Revolución Francesa, con los trabajo de J.C. Borda y N. de Condorcet y en relación con los sistemas de votación y sus eventuales inconsistencias. El enfoque moderno surge -como es conocido- de la obra de K.J. Arrow de 1951. Ahora bien, como destaca Sen, la teoría tradicional del bienestar desarrollada por F.Y. Edgeworth, A. Marshall y A.C. Pigou -entre otros- siguió una orientación distinta pues se basó en el cálculo utilitarista de J. Bentham centrado en el cálculo de la utilidad total, prescindiendo de los aspectos distributivos. La teoría del bienestar, a partir de los años cuarenta utilizó únicamente el criterio de Pareto con algunas novedades, elaboradas por P.A. Samuelson y A. Bergson y, especialmente, la ya mencionada aportación de Kenneth J. Arrow que inauguró la teoría del social choice basada en una metodología axiomática. Sen subraya que en dicho marco los problemas distributivos del tipo reparto del pastel raramente tienen solución consistente; en rigor, los procedimientos de votación son completamente naturales para ciertos tipos de elecciones sociales, como es el caso de las elecciones, los referendos o las decisiones de los comités pero, por el contrario, son totalmente inadecuados para muchos otros problemas de elección social. Sen sostiene claramente que para analizar cuestiones relacionadas con la distribución es necesario ir más allá de las reglas de votación. Así, escribe:

Para establecer los fundamentos de una teoría constructiva de la elección social, si deseamos rechazar el consenso histórico contrario al uso de comparaciones interpersonales en la elección social, entonces precisamos enfocar dos importantes -y difíciles- cuestiones, La primera es ¿podemos incorporar y utilizar algo tan complejo como las comparaciones interpersonales relativas a un elevado número de personas?... La segunda es ¿cómo pueden ser integrados los resultados analíticos en un uso práctico? (1999a, 188)

Sobre la primera cuestión sostiene que "las comparaciones interpersonales de diversos tipos pueden ser completamente axiomatizadas e incorporadas con exactitud en los procedimientos de elección social -mediante el uso de 'condiciones de invariancia' en una estructura generalizada, formalmente construida como "funcionales de bienestar social"” (1999a, 188), tema sobre el que cita sus propias aportaciones-. Y se pregunta hasta qué punto los resultados del tipo "imposibilidad de Arrow" desaparecen cuando se opera con comparaciones interpersonales de bienestar; afirma que desaparecen efectivamente como consecuencia de la disponibilidad de más información relevante. Volverá sobre ello.

La segunda cuestión, relativa a la base informativa, surge de preguntarse sobre qué dimensiones queremos efectuar comparaciones interpersonales. Una primera opción sería referirse a los estados mentales de felicidad pero Sen destaca que existen razones para descartarla. Otra opción sería utilizar los bienes primarios de Rawls o referirse a las necesidades básicas; o bien emplear el enfoque propuesto en los Human Development Reports del "United Nations Development Program", en cuyo diseño participó él mismo. Sen trata asimismo el tema de la pobreza, como ilustración de los problemas que plantea la dimensión informativa y analiza asimismo brevemente el tema de las privaciones comparativas y de la desigualdad de género.

En las observaciones finales, Sen reafirma sus ideas sobre la posibilidad de desarrollar una economía del bienestar y una teoría de la elección colectiva constructivas, ampliando sus bases de información; en especial ampliando su uso en las comparaciones interpersonales y sin olvidar, por otra parte, la profunda complementariedad existente, al tratar el tema, entre el razonamiento formal, matemático, y el informal.

\section{Teoría del desarrollo: Desarrollo y libertad}


La teoría del desarrollo constituye un tema central de la obra de Amartya Sen. Su libro de síntesis Development as Freedom, ${ }^{17}$ no obstante, no fue publicado hasta 1999 , precedido de un artículo en el que efectúa un recorrido por las teorías existentes; ${ }^{18}$ Sen manifiesta que ha tratado de hacer su análisis lo menos técnico posible y explica que el origen de su texto se halla en cuatro conferencias pronunciadas en el Banco Mundial.

En la introducción Sen indica que en su enfoque: "la expansión de la libertad es tanto el fin primordial del desarrollo como su medio principal. El desarrollo consiste en la eliminación de algunos tipos de falta de libertad que dejan a los individuos pocas opciones y escasa oportunidades de para ejercer su agencia razonada. La eliminación de la falta de libertades fundamentales -es lo que sostenemos aquí- es una parte constitutiva del desarrollo" (2000 [1999], p. 16). El libro trata exclusivamente de los países en vías de desarrollo y -como veremos- lo hace desde una perspectiva singular, indicada en su título.

En la introducción Sen precisa que el desarrollo puede concebirse como un proceso de expansión de las libertades reales de las que disfrutan los individuos. El crecimiento del PNB o de las rentas personales constituye un medio importante para ello pero, al mismo tiempo, el desarrollo exige la eliminación de las principales fuentes de privación de la libertad, que son la pobreza y la tiranía. La libertad es fundamental -destaca Sen- por dos razones distintas, porque: (1) es el principal criterio de evaluación de los resultados obtenidos y (2) porque el desarrollo depende totalmente de lo que Sen denomina la libre agencia de los individuos, es decir, el hecho de que las libertades forman parte de los principales medios del desarrollo. De acuerdo con dicho enfoque, la libertad para realizar intercambios y transacciones forma parte de las libertades básicas y contribuye al desarrollo, sin que ello signifique excluir el papel de la ayuda social o las intervenciones públicas. Sen señala asimismo el papel de los valores sociales y las normas compartidas y distingue cinco tipos de libertades, desde una perspectiva instrumental: "(1) las libertades políticas; (2) los servicios económicos, (3) las oportunidades sociales, (4) las garantías de transparencia y (5) la seguridad protectora".

Precisa que la concepción de la libertad que ha adoptado "incluye tanto los "procesos' que hacen posible la libertad de acción y de decisión como las 'oportunidades' reales que tienen los individuos, dadas sus circunstancias "personales y sociales"' (2000 [1999], p. 33). Por otra parte -y a otro nivel- incluye explícitamente la libertad de realizar transacciones en los mercados como un aspecto de las libertades. En el capítulo siguiente distingue cinco aspectos relacionados con la eficacia instrumental de las libertades, en particular (1) las libertades políticas; (2) los servicios económicos, (3) las oportunidades sociales, (4) las garantías de transparencia y (5) la seguridad protectora. Sen expone seguidamente sus posiciones sobre las bases informativas de la justicia, el utilitarismo, el pensamiento libertario, Rawls, Nozick y otros pensadores. En la última parte del capítulo se centra en analizar las diversas fuentes de diferencias entre nuestra renta real y las ventajas, en términos de bienestar y libertad, que extraemos de ella.

Sen identifica y analiza las siguientes fuentes: (1) la heterogeneidad personal; (2) la diversidad relacionada con el medio ambiente; (3) las diferencias de clima social; (4) las diferencias entre las perspectivas relacionales, y (5) la distribución de recursos y libertades en el seno de la familia (2000 [1999], pp. 94-96). Argumenta que la renta constituye una guía limitada para evaluar la calidad de vida y somete a crítica la idea muy extendida de que la pobreza se reduce a escasez de renta e indica que tampoco es suficiente la consideración de los bienes primarios de John Rawls; finalmente, analiza el tema desde su propia perspectiva de las capacidades, que ya hemos considerado.

El siguiente capítulo está dedicado a la pobreza como privación de capacidades que constituye el enfoque de Sen para tratar el tema. La relación entre la renta y las capacidades varía mucho según la edad, el sexo, los papeles sociales o los lugares pues estas circunstancias pueden exigir una renta mayor para conseguir las mismas funciones; debido a ello la eliminación de la

\footnotetext{
${ }^{17}$ Sen A. 1999. Development as Freedom. New York, Oxford University Press. Edición española de 2000.

${ }^{18}$ Sen A. 1997. Traducido en Sen, A. 1998. Las teorías del desarrollo a principios del siglo XXI, Cuadernos de Economía XVII(29), Bogotá, 73-100.
} 
pobreza de renta no puede ser, simplemente, el objetivo. Dedica una especial atención al fenómeno del paro, especialmente en sus vertientes que van más allá de la pérdida de ingresos daños psicológicos, pérdida de confianza, deterioro de la salud, etc.-. Analiza de modo especial las consecuencias del paro en diversos grupos raciales en los Estados Unidos, un análisis que le permite confirmar la pertinencia de su enfoque. Los casos extremos del sur de Asia y del África subsahariana confirman también su aproximación al tema. Dedica una especial atención a la alta mortalidad y a las bajas tasas de supervivencia de las mujeres en diversas partes del mundo que reflejan una elevada privación de sus capacidades básicas.

Sen analiza seguidamente la relación existente entre los mercados, el Estado y las oportunidades sociales. Defiende las libertades que aportan los mercados, lejos de las restricciones características de la servidumbre; destaca que el debate sobre la eficiencia de los mercados deja abierto el tema de la equidad de la distribución resultante y afirma que ésta es preciso considerarla no sólo en términos de renta sino también en términos de las libertades y de las capacidades fundamentales. Se plantea asimismo los límites del mecanismo de mercado en relación con los bienes públicos, especialmente en el campo del medio ambiente y de la sanidad, destacando que pueden existir razones para proporcionar bienes públicos, especialmente en los campos de la educación básica y los servicios sanitarios y otros bienes públicos (o semipúblicos), de entre los que también cita la defensa, la policía y la protección del medioambiente. Plantea asimismo el tema de los incentivos y los problemas asociados a la selección de los destinatarios de las ayudas.

La democracia -afirma Sen- no es un lujo para los países pobres; antes al contrario, constituye una necesidad y ello es así debido a tres consideraciones distintas que indican la primacía general de los derechos políticos y liberales básicos:

(1) La importancia "directa" en la vida humana relacionada con las capacidades básicas -incluida la participación política y social-; (2) su papel "instrumental” en la mejora de las posibilidades de los individuos para expresar y defender sus demandas de atención política -incluidas sus exigencias de que se satisfagan sus necesidades económicas-; (3) su papel "constructivo" en la conceptualización de las necesidades (incluida la comprensión de las necesidades económicas en un contexto social). (2000 [1999], p. 185)

Se plantea si el autoritarismo proporciona buenos resultados. Su posición es clara y afirma que "apenas existen pruebas de que el régimen autoritario y la supresión de los derechos políticos y humanos contribuyan en realidad a fomentar el desarrollo económico" (2000 [1999], p. 187).

Todo un capítulo está dedicado a las hambrunas y otras crisis. Sen ha analizado reiteradamente el desarrollo de las hambres súbitas -las hambrunas- que no se explican únicamente por una reducción súbita de la oferta de alimentos sino que, con alta frecuencia, se explican por el funcionamiento de la economía y de la sociedad en general y, en especial, por la falta de derechos económicos y diversos comportamientos perversos. Un régimen democrático, por otra parte, considera que genera las condiciones institucionales que inducen a una intervención eficaz para prevenir y mitigar las hambrunas y otras crisis súbitas.

El capítulo siguiente está dedicado a la agencia de las mujeres y el cambio social. Como ya hemos visto, la consideración de lo que denomina la agencia de las mujeres va más allá de prestar atención a su bienestar propio para centrarse además en su papel como protagonistas, como agentes activos. Su planteamiento incluye temas como la resolución de conflictos mediante la cooperación y afecta desde la fecundidad a la distribución de los alimentos y la educación, al cuidado de los hijos y de los mayores, la salud y el empleo, entre otros aspectos importantes que incluyen también su papel social, económico y político y en los que las mujeres sufren discriminación de género. Sen considera esta dimensión un aspecto muy importante del desarrollo como libertad.

Se trata de una dimensión incorporada en diversos trabajos de la economía feminista; véase, por ejemplo, el artículo de Lourdes Beneria -expresidenta de International Association of Feminist Economics: "De la 'armonía' a los 'conflictos cooperativos"' (2008). Por otra parte, el libro de B. Agarwal, J. Humphries e I. Robeyns: Amartya Sen's work and ideas: a gender pers- 
pective (2005) contiene una discusión sistemática del tema de la dimensión del género en la obra de Sen; la entrevista o diálogo con Sen resulta altamente clarificadora.

Sen analiza los problemas de la nutrición a escala mundial y considera existen pocas pruebas de que -a dicha escala- la producción de alimentos crezca menos que la población; la tendencia de la producción de alimentos es ascendente. En este contexto, analiza la aceptabilidad de la solución coercitiva para reducir el crecimiento de la población y para reducir las tasas de fecundidad; considera que "el enfoque que parece merecer especial atención entraña la existencia de una estrecha relación entre las medidas que aumentan la igualdad sexual y la libertad de las mujeres [...] y la responsabilidad social de la familia" (2000 [1999], p. 273).

Sobre la elección social y la conducta individual destaca que la idea de utilizar la razón para identificar y promover las sociedades mejores y más aceptables ha sido una poderosa fuerza impulsora colectiva en el pasado y continúa siéndolo hoy día; ahora bien, para aplicar la razón, es preciso disponer de metodologías de evaluación así como de instituciones adecuadas; en este contexto indica que el "teorema de imposibilidad de Arrow" invita a destacar, precisamente, la necesidad de aumentar las bases de información utilizadas para poder hallar mecanismos de elección que proporcionen efectivamente soluciones; subraya asimismo que las reglas decisión por mayoría no pueden servir para resolver los conflictos económicos.

Contempla de nuevo el tema de la motivación humana y analiza el papel de los valores en el capitalismo, destacando que éste no puede funcionar únicamente con codicia pues necesita la confianza mutua y el respecto de ciertas normas; ilustra el tema mediante las dificultades de funcionamiento en la antigua Unión Soviética y de los países del Este de Europa; lo ilustra asimismo con la consideración de la Mafia y sus reglas propias y, finalmente, con un análisis de la corrupción.

El último capítulo se titula "La libertad individual como un compromiso social". Sen sostiene que un elemento fundamental para hacer frente a los retos del mundo contemporáneo es la idea de lo que constituye una sociedad aceptable y que, en su caso, ha defendido la primacía de las libertades fundamentales como criterio para la evaluación de las ventajas individuales y de los éxitos y los fracasos sociales. Se trata de un enfoque que puede generar una idea del desarrollo distinta de la que se concentra en las magnitudes dinerarias y que -contrariamente-conduce a dar importancia a problemas como la pobreza y las desigualdades desde una perspectiva coherente.

El libro trata exclusivamente el problema del desarrollo de los países en vías de desarrollo y como hemos visto- lo hace desde una perspectiva singular, de modo que el lector hallará escasas -sino nulas- referencias a temas más convencionales, como el papel del Estado en sectores económicos básicos como la siderurgia o las infraestructuras de transporte o bien las inversiones en I+D o el papel de los diversos tipos de innovaciones -especialmente tecnológicas-, o las funciones asignadas -o no- a la inversión extranjera.

Mercados y planificación. El ya mencionado artículo -contemporáneo del libro Development and Freedom sobre el pensamiento económico relativo al desarrollo- Sen expresa opiniones sobre mercado e intervención estatal que es útil recoger como síntesis de sus posiciones.

Así, Sen precisa que "el hecho de reconocer las virtudes del mercado no debe inducirnos a ignorar las posibilidades y los logros ya constatados del Estado o, por el contrario, a considerar al mercado como factor de éxito independientemente de toda política gubernamental". ${ }^{19}$ Prosigue señalando los límites de las formulaciones que priorizan radicalmente la acumulación y se desinteresan por la mejora de las condiciones de vida existentes y afirma asimismo que no existen estudios estadísticos sistemáticos que corroboren la existencia de un enfrentamiento entre los derechos políticos y la actividad económica. Finalmente, analiza y critica las orientaciones basadas en el concepto de capital humano para centrarse en sus posiciones relativas a la expansión de las capacidades.

A mi juicio, existe un aspecto a destacar de la concepción de Sen relativa al papel de los mercados, consistente en la práctica ausencia de referencias al papel de las innovaciones propulsa-

\footnotetext{
${ }^{19}$ Pp. 4-5 la citada traducción de 1998 en Cuadernos de Economía, XVII(29).
} 
das por el sector privado de la economía y en el marco de las políticas encaminadas a propiciar un proceso de desarrollo.

\section{El desarrollo y la información estadística}

La concepción de Sen sobre el desarrollo y las capacidades, así como su preocupación por la información, tuvo una interesante concreción con la implementación de su propuesta de un Índice de Desarrollo Humano por parte de la Naciones Unidas a partir de 1990 y que ya hemos comentado anteriormente. ${ }^{20}$ Por otra parte, en 2010 apareció el libro, obra de Amartya Sen, Joseph Stiglitz y Jean Paul Fitoussi, Mismeasuring our lives: why GDP doesn't add up: the report (New York-London: New Press). ${ }^{21}$ Constituye el informe final de la comisión dirigida por los tres autores y creada a instancias del presidente francés Nicolas Sarkozy con la finalidad de evaluar la pertinencia y la adecuación de las estadísticas del Producto Interior Bruto monetario como instrumento para evaluar el bienestar y el progreso, concebidos multidimensionalmente, así como para formular propuestas de mejora y para su adecuada medición.

La medición del bienestar multidimensional exige considerar magnitudes e indicadores en términos monetarios como renta, consumo, riqueza pero también otros relativos a salud, educación, condiciones de trabajo, seguridad y medio ambiente, por citar sólo algunos ejemplos.

Existen propuestas muy serias, en especial: Comisión de las Comunidades Europeas, Más allá del PIB. Evaluación del progreso en un mundo cambiante, basado en el Informe StiglitzSen-Fitousssi (2008): Commission on the Measurement of Economic Performance and Social Progress.

\section{La idea de justicia}

Sen publicó en 2010 La idea de justicia $;^{22}$ en el prefacio explica que su propósito no es formular una teoría de la justicia en el sentido de determinar las instituciones perfectamente justas sino poder realizar comparaciones pertinentes en términos de justicia. Para poder efectuar lo primero es preciso formular unos principios generales de justicia y precisa que pueden existir serias diferencias entre los principios rivales de justicia surjan del análisis crítico y que pretendan imparcialidad; con ello anticipa este problema en relación con las posiciones de John Rawls. Por el contrario, si el objetivo es básicamente orientar la elección razonada de políticas, estrategias o instituciones, entonces la identificación de esquemas sociales completamente justos no resulta necesaria ni suficiente. Conviene recordar que, como hemos visto ya en secciones anteriores, Sen se ha ocupado en diversas ocasiones, precisamente de la valoración y comparación de alternativas.

Rawls y la justicia como equidad. Sen utiliza la frase "El razonamiento buscado al analizar los requisitos de la justicia incluirá algunas exigencias básicas de imparcialidad" (2010, p. 71) para introducir la obra de Rawls titulada La justicia como equidad, puesto que la equidad guarda relación con la imparcialidad. El capítulo 2 está dedicado a la obra de Rawls, una de las más influyentes sobre el tema -si no la que más- y de la que Sen se ha ocupado en numerosas ocasiones. Para no resultar críptico convendrá formular unas precisiones introductorias sobre la posición de Rawls. ${ }^{23}$

Rawls indica que "La idea más fundamental en esta concepción de la justicia es la idea de la sociedad como un sistema equitativo de cooperación social a lo largo del tiempo de una generación a la siguiente. Usamos esta idea como la idea organizadora central cuando tratamos de desarrollar una concepción política de la justicia para un régimen democrático" (Rawls 2002 [1999], p. 28). Seguidamente, Rawls formula dos ideas fundamentales adicionales, relacionadas entre sí: la idea de los ciudadanos como personas libres e iguales y la idea de sociedad bien

\footnotetext{
${ }^{20}$ Véase http://hdr.undp.org/es/content/el-\%C3\%ADndice-de-desarrollo-humano-idh

${ }^{21}$ Traducción al castellano: Sen, A. 2013. Medir nuestras vidas. Barcelona, RBA libros.

${ }^{22}$ Sen A. 2010. The Idea of Justice. London, Penguin. Edición española: 2010. Teoria de la justicia. Madrid, Taurus.

${ }^{23}$ Véase Vegara, J.M. 1.7.2013. Los valores de la izquierda y los obstáculos, L'opinió, Revista de la Fundació Rafael Campalans.
} 
ordenada, es decir, de una sociedad regulada de modo efectivo por una concepción política de la justicia, de la que él se ocupará.

Su idea de justicia se refiere para Rawls a la estructura básica de una sociedad bien ordenada Por otra parte precisa que "No concebimos la justicia como equidad como una doctrina moral comprehensiva sino como una concepción política que se aplica a esa estructura de instituciones políticas y sociales" (Rawls 2002 [1999], p. 36); así pues, no propone una idea de justicia con pretensiones éticas de validez en todos los campos de la vida humana sino -más limitadamenteuna propuesta de justicia relevante para la vida política y sus instituciones. Nuestro autor precisa asimismo que "nos preguntamos cómo podría ser un régimen constitucional perfectamente justo o cuasi justo, y si podría tener lugar y hacerse estable bajo las circunstancias de la justicia, es decir, bajo condiciones realistas aunque razonablemente favorables" (Rawls 2002 [1999], p. 36). Resulta claro pues que el campo al que Rawls se limita tiene un carácter normativo y relativo a un régimen constitucional perfectamente justo, o cuasi- justo.

En el marco acotado por los límites indicados, Rawls se pregunta:

Supongamos, en particular, que las desigualdades sociales y económicas fundamentales son las diferencias en las perspectivas vitales de los ciudadanos (las perspectivas sobre su vida entera), en la medida en que son afectadas por cosas tales como su clase social de origen, sus dotaciones innatas, sus oportunidades de educación y su buena o mala fortuna a lo largo de toda su vida. Preguntamos imediante qué principios se legitiman las diferencias de esta clase -diferencias en las perspectivas vitalesy se hacen consistentes con la idea de una ciudadanía libre e igual en una sociedad concebida como un sistema equitativo de cooperación? (Rawls 2002 [1999], p. 70)

Considero pertinente destacar que Rawls se plantea la cuestión de cómo pueden justificarse diferencias y desigualdades y para responder utiliza la idea de la posición original y de velo de la ignorancia que juegan un papel clave en su concepción (Rawls 2002 [1999], p. 38).

La equidad y el velo de la ignorancia. Rawls concibe pues la justicia como equidad y ésta como una exigencia de imparcialidad, consistente en no dejarse influir por los propios intereses; por ello, en su formulación de los "Principios que definen una sociedad justa" juega un papel importante la noción de velo de la ignorancia o la posición original, es decir, "una situación imaginaria de igualdad primordial, donde las partes no tienen conocimiento de sus identidades reales ni de sus intereses creados en el grupo como tal. Sus representantes tienen que escoger bajo el 'velo de la ignorancia', que es un estado imaginario de ignorancia para decidir -en particular, ignorancia acerca de los distintos intereses personales y las diferentes opiniones reales sobre la 'buena vida' (lo que Rawls llama 'preferencias comprehensivas')-" (Rawls 2002 [1999], p. 83).

En otras palabras, los representantes de los ciudadanos sujetos sometidos al velo de la ignorancia de Rawls participan en una situación imaginaria en la que ignoran las características generales de la sociedad y las personales de todos los participantes, incluidos ellos mismos. De este modo se aseguraría que elegirán unos principios de justicia prescindiendo de sus propias situaciones e intereses personales -que les podrían asegurar posiciones favorables o desfavorables- y resultantes de la suerte, sea ésta producto del azar natural -su raza, su sexo o su inteligencia innata, por ejemplo- o los resultantes de sus circunstancias sociales -su clase social de pertenencia, su renta o la educación recibida-. El procedimiento está diseñado pues para garantizar la imparcialidad.

La elección de los principios básicos de justicia es el primer acto en el despliegue de la justicia social; en la siguiente se seleccionan instituciones. Sen subraya -punto básico que anticipo aquí- que Rawls considera que de este proceso sólo puede resultar una elección única, un punto básico del que Sen discrepará con energía.

Los bienes primarios. Un primer resultado del proceso de la posición original es la formulación de cinco clases de lo que Rawls denomina bienes primarios que las personas necesitan como ciudadanos. Concretamente son: 
i) Los derechos y libertades básicos: la libertad de pensamiento y la libertad de conciencia, juntamente con las demás [...] ii) La libertad de movimiento y la libre elección de empleo en un marco de oportunidades variadas que permitan perseguir diversos fines y que dejen lugar a la decisión de revisarlos y alterarlos. iii) Los poderes y las prerrogativas que acompañan a cargos y posiciones de autoridad y responsabilidad. iv) Ingresos y riqueza, entendidas ambas cosas como medio de uso universal (con un valor de cambio) que suelen necesitarse para lograr un amplio abanico de fines, cualesquiera sean éstos; y v) Las bases sociales del autorespeto, con lo que entendemos aquellos aspectos de las instituciones básicas normalmente esenciales si los ciudadanos han de tener clara conciencia de su valor como personas y han de ser capaces de promover sus fines con autoconfianza. (Rawls 2002 [1999], pp. 91-92)

Como observará el lector, los bienes básicos no son únicamente ingresos y riqueza; son más amplios.

Los dos principios de Rawls. Seguidamente Rawls enuncia los dos principios de la justicia que, en su reformulación de 2001 son (Rawls 2002 [1999], p. 73):

- Principio1: cada persona tiene el mismo derecho irrevocable a un esquema plenamente adecuado de libertades básicas iguales que sea compatible con un esquema similar para todos.

- El Principio 2 lo denomina:

Principio de diferencia: Las desigualdades sociales y económicas tienen que satisfacer dos condiciones: en primer lugar tienen que estar vinculadas a cargos y posiciones abiertos a todos en condiciones de igualdad equitativa de oportunidades $\mathrm{y}$, en segundo lugar, las desigualdades deben redundar en un mayor beneficio de los miembros menos aventajados de la sociedad (el principio de diferencia). (Rawls 2002 [1999], p. 73)

Los dos principios están ordenados en su prioridad relativa: el primer principio precede al segundo y dentro del segundo principio la igualdad equitativa de oportunidades tiene precedencia sobre el principio de diferencia. Una libertad básica tan solo se puede limitar en beneficio de otra libertad básica, nunca en beneficio de una mejora de las condiciones sociales y económicas. El primer principio suscita generalmente pocas discrepancias; el segundo principio -de la diferencia- es más discutido. Este principio permite la existencia de desigualdades sociales y económicas asociadas a funciones necesarias siempre que aporten beneficios a aquéllos que se hallan en la situación más desfavorable, es decir, que los incentivos operen de modo eficaz para ello. Para cumplir este segundo principio Rawls sostiene que:

Deben imponerse ciertos requisitos a la estructura básica [de la sociedad], requisitos más exigentes que los de los sistemas de libertad natural. Un sistema de libre mercado debe establecerse en un marco de instituciones políticas y legales que ajuste la tendencia a largo plazo de las fuerzas económicas a fin de prevenir las concentraciones excesivas de propiedad y riqueza, especialmente de aquéllas que conducen a la dominación política. (Rawls 2002 [1999], p. 74)

Como se observará, Rawls expresa qué puede justificar -según su formulación- determinadas diferencias y desigualdades, puesto que las desigualdades asociadas a los incentivos considera tienen límites.

La discrepancia clave. Sen coincide con Rawls en que la dimensión de la equidad es central para definir la justicia pero -por su parte- no considera que la imparcialidad generada a partir de la posición original resulte válida: Sen discrepa de Rawls cuando éste afirma que del proceso del velo de la ignorancia sólo pueden surgir unos principios de justicia únicos, precisamente los que el propio Sen enuncia y argumenta a lo largo del libro.

La segunda discrepancia guarda relación con la identificación de con quiénes tenemos obligaciones derivadas de la justicia. Sen señala aquí tres problemas distintos.

En primer lugar, subraya que la justicia es en parte una relación en la que la idea de obligación con el otro es importante y, por ello, resulta clave determinar qué personas forman parte de estos otros. De esta consideración surge el tema de la justicia en relación con los otros, precisamente en el caso en que no pertenecen a un mismo Estado soberano y que, por ello, no pueden 
incluirse en el esquema de Rawls, centrado en las instituciones. La crítica de Sen es clara: "una teoría de la imparcialidad confinada exactamente dentro de las fronteras de un Estado soberano procede a lo largo de líneas territoriales que tienen, por supuesto, significación legal pero que pueden no tener relevancia política o moral" (Sen 2010, p. 158)

En segundo lugar, Sen subraya que -por otra parte- existen acciones de un país que pueden afectar seriamente a otras vidas en otros países y que las instituciones de uno de ellos pueden afectar seriamente a otros países, no sólo mediante las relaciones económicas sino incluso mediante el uso de la fuerza; Sen pone como ejemplo la invasión norteamericana de Irak en 2003 (Sen 2010, p. 159).

Y finalmente, Sen destaca una dimensión adicional relevante que deriva del hecho de que "la evaluación de la justicia exige compromiso con "'los ojos de la humanidad'; primero, porque podemos identificarnos con los otros y no sólo con nuestra comunidad local; segundo, porque nuestras opciones y acciones pueden afectar las vidas de otros aquí y allá; y tercero, porque lo que los otros ven desde sus perspectivas históricas y geográficas puede ayudarnos a superar nuestro propio parroquialismo" (Sen 2010, p.159).

Rawls plantea, como solución del tema, precisamente una segunda posición original entre los representantes de diferentes comunidades para definir los principios de la justicia internacional (Rawls 2002 [1999], p. 169-174); la dificultad de este enfoque radica en la falta de realismo que supone considerar que - a diferencia de los que ocurre en el seno de un Estado soberano- es viable instaurar las instituciones necesarias para aplicar los principios enunciados.

La tercera discrepancia de Sen surge al contraponer su concepción de las capacidades a la los bienes básicos de Rawls. Previamente destaca que si bien Rawls propone correctivos para tener en cuenta necesidades especiales como las que surgen de discapacidades y de diversas desventajas, ello no deriva propiamente de sus principios de justicia pues, como señala Sen, dichas "correcciones no llegan con el establecimiento de la 'estructura institucional básica' de la sociedad en la 'fase constitucional', sino que aparecen más tarde con ocasión del uso de las instituciones ya establecidas, particularmente en la 'fase legislativa"' (Rawls 2002 [1999], pp. 290-291). No son pues rasgos fundamentales de la concepción de Rawls. Como hemos examinado ya, Sen considera que lo adecuado es referirse a capacidades y no a los bienes primarios de Rawls. En el libro de Sen este enfoque -que pone el acento en las oportunidades reales disponibles más que en los medios disponibles- es extensamente expuesto en los capítulos 11 y 12; en éste último, Sen analiza de nuevo la pobreza como privación de capacidad.

¿Igualdad de capacidades? Sen prosigue analizando las relaciones existentes entre felicidad, bienestar y capacidades y se plantea precisamente si "la igualdad es importante, y la capacidad constituye, en efecto, un rasgo central de la vida humana (como he tratado de sostener en este libro), ¿no sería justo suponer que debemos exigir igualdad de capacidad?", a lo que responde negativamente por varias razones (Sen 2010, p. 325). La primera razón consiste en que "la capacidad $[\ldots]$ no puede prestar adecuada atención a la rectitud y la equidad implicada en los procedimientos que tienen relevancia para la idea de la justicia" (Sen 2010, p. 325). La segunda razón está relacionada con otras exigencias que ejemplifica con los esfuerzos y las recompensas que deben asociarse al trabajo. La tercera razón está relacionada con la ponderación de las diversas capacidades, o sea, con la elección de las ponderaciones que deben asignarse a los diferentes tipos de capacidades o de actividades, de modo que resulta importante para apreciar los límites de la relevancia de la igualdad de capacidad como parte de las exigencias de justicia. Y la cuarta razón consiste en que la igualdad no es en sí misma el único valor relevante para una teoría de la justicia; así, por ejemplo, una política puede ser defendida porque mejora las capacidades de todos aunque no mejore su distribución.

Aunque pueda sorprender, la no asunción de la igualdad de capacidades como criterio de justicia no supone un cambio de posición pues ya en la denominada "Tanner Lecture" de 1979 "Equality of What?"- Sen ya había propuesto su enfoque basado en las capacidades básicas pero consideraba éstas como "una guía parcial de la parte de la bondad moral que se halla asociada con la idea de igualdad. Y he tratado de argumentar que como un guía parcial posee las virtudes de las que carecen las otras características de la igualdad" (Sen 1982, p.369). Existe pues continuidad en su posición. En este contexto considero pertinente subrayar que, al centrarse Sen en las capacidades básicas, de ello deriva que su enfoque sería insuficiente para evaluar la justicia 
en las sociedades desarrolladas pues -a pesar de que en éstas no se hallen cubiertas dichas capacidades básicas para todos los ciudadanos- las diferencias y las desigualdades existentes no se refieren únicamente a niveles básicos sino que van más allá, dado los niveles medios de bienestar alcanzados; parece pues indispensable elaborar criterios que incluyan dimensiones comparativas relativas a niveles no básico o bien un nuevo enfoque adaptado a una mayor complejidad.

El debate reciente sobre la India. La obra de Sen está centrada claramente en los problemas existentes en los países en vías de desarrollo y, en especial, en los problemas del desarrollo de la India, como puede comprobarse en su reciente libro escrito con Jean Drèze, An Uncertain Glory (2014 [2013]), y que contiene una interesante síntesis, muy detallada -relativa al caso de la India- de su concepción del desarrollo y de sus posiciones políticas. La obra ha suscitado una gran polémica, en especial con el también economista indio -profesor en la Universidad de Columbia- Jagdish Bhagwati, autor principal de Why Growth Matter ${ }^{24}$ y también muy activo, en años anteriores, en la política económica de la India; los dos libros fueron publicados en 2013 y la polémica ha tenido un amplio reflejo académico pero también político y periodístico.

Sen y Drèze señalan las limitaciones de las realizaciones de la democracia india conseguidas después de la independencia pero critican, especialmente, el giro liberal de la política económica implementado por la nueva mayoría política vigente en la India desde 1991 y cuyos resultados evalúan muy críticamente.

Las dos obras manifiestan profundas discrepancias sobre la situación real y su evolución. En su diagnóstico, los autores de An Uncertain Glory aplican el esquema analítico de Sen basado en las capacidades y la agencia y analizan con detalle las desigualdades de clase, casta y género, especialmente en términos de educación, sanidad y nutrición; prestan también una notable atención a los problemas del medio ambiente y denuncian la mejora de la situación de los sectores minoritarios privilegiados así como la política de subsidios a los grandes consumidores. Su diagnóstico difiere notablemente del formulado por Bhagwati y Panagariya.

Es destacable que los autores de An Uncertain Glory denuncien lo que califican de la extensa corrupción existente en el país pero sitúen sus orígenes en la denominada "licencia Ra", implantada y mantenida por los gobiernos anteriores y necesaria para iniciar cualquier actividad económica privada; califican el tema de la corrupción de problema endémico dels país.

Un punto clave del enfrentamiento se halla relacionado -lógicamente- con el papel y la importancia de la redistribución, orientada, en las propuestas de Sen y Drèze, según el enfoque del desarrollo de las capacidades y concebida como aplicada simultáneamente con el impulso del desarrollo económico. Propugnan la continuidad de la Ley de Garantía del Empleo Rural de 2005 que permite financiar actividades que generan empleo mediante obras públicas, así como también el Sistema Público de Distribución, una modalidad de racionamiento selectivo. En términos generales defienden amplias políticas redistributivas centradas en la educación, la sanidad y la nutrición, generalmente en términos no monetarios, al contrario de lo que propugna Bhagwati.

Es destacable que el libro de Sen y Drèze no contiene una propuesta global sobre las reformas y las políticas económicas sectoriales que deberían contribuir a generar los recursos necesarios para financiar las políticas específicas que propugnan: me refiero, por ejemplo, a la política de infraestructuras, la política agraria o la industrial; posiblemente ello sea debido a que prefieren concentrarse en la explicación de las características singulares de su concepción del desarrollo pero, no obstante, resulta inevitable que el lector se pregunte por dicha ausencia.

\section{A modo de conclusión}

La extensa obra de Sen ocupa un merecido y relevante lugar en el campo de la economía política actual y que ha dado lugar, también, a una notable polémica que incluye el campo de la filo-

Bhagwati, J. y Panagarya, A. (2013). Véase la evaluación que realizaba Bhagwati cuatro décadas antes de la experiencia india en Desai P., Bhagwati J. 1975. Socialism and Indian Economic Policy, World Development, 3(4), April, 213-221. 
sofía política. Premio Nobel de Economía por sus contribuciones a la economía del bienestar, Sen ha sido catedrático en numerosas Universidades de prestigio y ha presidido también diversas Asociaciones de economistas; asimismo ha influido en diversas instituciones internacionales como el Banco Mundial o diversas agencias de las Naciones Unidas. Por otra parte, existen asociaciones profesionales como The Human Development and Capabilities Association, ampliamente inspiradas en su obra.

Su formación filosófica le permite tratar con rigor temas inhabituales en los escritos de un economista o bien le facilita incorporar dimensiones o reflexiones filosóficas y éticas en su discurso económico; le permite hacerlo y también le induce a hacerlo, sin duda. Este hecho puede sorprender por cuanto es más habitual que la componente filosófica-ética -o ideológica, si se prefiere- se halle implícita, oculta en un relato de contenido aparentemente tan sólo analítico y neutral. Sen es mucho más transparente.

\section{Bibliografía}

Agarwal, Bina, Humphries, Jane y Robeyns, Ingrid. 2005. Amartya Sen's work and ideas: a gender perspective. London, Routledge.

Arrow, Kenneth J. 1974 [1951]. Elección social y valores individuales. Madrid, Instituto de Estudios Fiscales.

Arrow, Kenneth .J, Sen, Amartya y Suzumura, Kotaro. 2002. Handbook of Social Choice and Welfare. Amsterdam, North Holland, Elsevier.

Atkinson, Anthony B. 1999. The Contribution of Amartya Sen to Welfare Economics, Scandinavian Journal of Economics, 101(2), 173-190.

Basu, Kaushik y Kanbur, Ravi. 2009. Arguments for a Better World. Oxford, Oxford University Press, 2 vols.

Beneria, Lourdes. 2008. De la "armonía" a los "conflictos cooperativos". La contribución de Amartya Sen a la "Teoría de la unidad doméstica, Araucaria. Revista Iberoamericana de Filosofía, Política y Humanidades, 20, $2^{\circ}$ semestre.

Bhagwati, Jagdish y Panagarya, Arvind. 2013. Why Growth Matter: How Economic Growth in India Reduced Poverty and the Lessons for Other Developing Countries. New York, Public Affairs.

Dasgupta, Partha, Sen, Amartya y Marglin, Stephen A. 1972. Guidelines for Project Evaluation. New York, UNIDO, United Nations.

Desai, Meghnad. 2001. Amartya Sen's Contribution to Development Economics, Oxford Development Studies, 29(3), 213-223.

Desai, Meghnad y Sen, Amartya. 2000. Prospect Magazine, 20, July.

Desai, Meghnad y Bhagwati, Jagdish. 1975. Socialism and Indian Economic Policy, World Development, 3(4), April, 213-221.

Dobb, Maurice Herbert. 1997. Sen, A. En J. Eatwell, M. Milgate and P. Newman, (eds.). The New Palgrave: A Dictionary of Economics. London, MacMillan Press.

Drèze, Jean y Sen, Amartya. 2014 [2013]. Una gloria incierta. Madrid, Taurus.

- Hunger and public action. Oxford (England) and New York, Clarendon Press, Oxford University Press.

Hicks, John. 1962. Book Review. A. Sen, Choice of Techniques, The Economic Journal, 72(.286), June, 379-381.

McMurrin, Sterling M. (ed.). 1982. The Tanner Lectures on Human Values. En Choice, Welfare and Measurement, Cambridge, Massachussets, London, England, Harvard University Press, 353-369.

Morris, Christopher. (ed.). 2010. Amartya Sen. New York, Cambridge University Press.

Prendergast, R. 2005. The concept of freedom and its relation to economic development: a critical appreciation of the work of Amartya Sen, Cambridge Journal of Economics, November, 1145-1170.

Rawls, John A. 1988 [1971]. La justicia como equidad. Madrid, Tecnos.

— 2002 [1999]. La justicia como equidad. Una reformulación. Barcelona, Paidós.

Schor, Armand-Denis. 2009. Amartya Sen. Vie, ceuvres, concepts. Paris, Élipses Marketing éditions. 
Stern, Nicholas. 1989. The Economics of Development: A Survey, The Economic Journal, 99(397) September, 597-685.

Sen, Amartya. 1959. Why planning?

http://www.indiaseminar.com/2008/589/589 amartya_kr_sen.htm

- 1960. Choice of Techniques. An Aspect of the Theory of Planned Economic Development. Oxford, Basil Blackwell.

- 1966. Labour allocation in a cooperative Enterprise, Review of Economics Studies, 33, 361371.

- 1967. A Possibility Theorem on Majority Decisions, Econometrica 34, 491-499.

- 1970. The Impossibility of a Paretian Liberal, Journal of Political Economy, 78, JanuaryFebruary, 152-157.

- 1973. On Economic Inequality. Oxford, Clarendon Press and New York, Norton.

- 1974. On Some Debates in Capital Theory, Economica, 41(163), August, 328-335.

- 1976. Rational Fools: A Critique of the Behavioral Foundations of Economic Theory, Philosophy and Public Affairs, 6(4), 317-344.

- 1980. Equality of What? Oxford, University of Utah Press and The Cambridge University Press.

- 1981. Poverty and Famines. Oxford, Clarendon University Press.

- 1982. Choice, Welfare and Measurement. London, Harvard University Press, Cambridge Mass.

- 1984. Resources, Values and Development. Cambridge Massachusetts, London, Harvard University Press.

- 1985. Commodities and Capabilities. New Delhi, Oxford University Press.

- 1987. Sobre ética y economía. Madrid, Alianza editorial.

- 1993. Achievements and Limitations of the Market Mechanism in Promoting Individual Freedoms, Oxford Economic Papers, New Series, 45(4), October, 519-541.

- 1997. Development thinking at the beginning of the $21^{\text {st }}$ century. London, London School of Economics, DEDPS, 2. Suntory and Toyota International Centers for Economics and Related Disciplines.

— 1999 [1992]. Nuevo examen de la desigualdad. Madrid, Alianza Editorial.

- 1999a. Nobel Lecture. The Possibility of Social Choice, American Economic Review, 89, July,.349.378.

- 1999b. Merit and Justice. En Arrow, K., Bowles, S. y Durlauf, S. (eds.). Meritocracy and Economic Inequality. Princeton-New Jersey, Princeton University Press.

- 2000 [1999]. Desarrollo y libertad. Barcelona, Planeta.

- 2004. Rationality and Freedom. London, The Belknap Press of Harvard University Press, Cambridge Mass.

- 2006. Identity and Violence: The Illusion of Destiny. New York, W.W. Norton \& Co., Issues of our time.

— 2009. El capitalismo: Más allá de la crisis, Claves, 191, abril, 14-19.

- 2010. La idea de justicia. Madrid, Taurus.

- 2011. Peace and Democratic Society. Cambridge (UK), Open Book Publishers.

Sen, Amartya, Stiglitz, Joseph.E. y Fitoussi, Jean-Paul. 2013 [2010]. Medir nuestras vidas. Barcelona, RBA libros.

Sen, Amartya y Williams, Bernard. (eds.) 1982. Utilitarism and beyond. New York, Cambridge University Press y Paris, Éditions de la Maison des Sciences de l'Homme.

Smith, Adam. 2009 [1759] .Teoría de los sentimientos morales. Madrid, Alianza Editorial.

Streeten, Paul. 2000. Freedom and Welfare: A Review Essay on Amartya Sen, 'Development as Freedom', Population and Development Review, 26(1), March, 153-162.

VV. AA. 1999a [1998]. Les Prix Nobel. The Nobel Prizes. Stockholm, Tore Frängsmyr, Nobel Foundation

- 1999b [1998]. The Sveriges Riksbank Prize in Economic Sciences in Memory of Alfred Nobel. Singapore, New Jersey, London, Hong Kong, World Scientific. 
- 2013. Ideological Profiles of the Economics Laureates, Economic Journal Watch, 10(3), September, 604-616. 